\title{
Creating and Updating models of Activities for people with Alzheimer Disease
}

\author{
Using JClic platform
}

\author{
Lorenzo C. Gonzalez ${ }^{1}$, Muhammed A. Mashat ${ }^{2}$ \\ ${ }^{1}$ Information Systems Department, Faculty of Computing \\ and Information Technology \\ ${ }^{2}$ Medical Education Department, Faculty of Medicine \\ King Abdulaziz University (KAU) \\ Jeddah, Saudi Arabia \\ \{lgonzalez, mamashat\}@kau.edu.sa
}

\author{
Sebastian Romero Lopez \\ Information Systems Department \\ University of Castilla-La Mancha (UCLM) \\ Albacete, Spain \\ Sebastian.romero@gmail.com
}

\begin{abstract}
The development and inclusion of new technologies and touch screen devices in the medical field has facilitated the treatment and rehabilitation of different types of patient. For this paper, we propose the use of touch screen devices for the rehabilitation of people with brain diseases such as Alzheimer's or for people who have suffered a stroke. We have created different kinds of activity models for the JClic platform for different users. We will use the models to create exercises that such people can do in an easy, intuitive, and visual form, allowing the user to participate more dynamically in their treatment or recovery, and assisting the work of medical staff.
\end{abstract}

Keywords-rehabilitation; human computer interaction; activities; models; JClic.

\section{INTRODUCTION}

The rehabilitation of people with neurological problems, whether due to diseases such as Alzheimer or a stroke, can be difficult without the right tools and a positive attitude from the rehabilitator [1]. If we do not have such tools, the progress of rehabilitation is much slower, causing some frustration in the patient, family and the person in charge of the treatment. Without a good attitude towards rehabilitation by the person who is in charge of that function, the recovery time or the maintenance of the patient is much higher, and in the worstcase scenario, the rehabilitation is ineffective.

To improve the current rehabilitation process and assist the rehabilitation of patients, we are going to use the JClic platform [2]. We have changed its parameters and created new activity models to suit the needs of users. There will be a visual, interactive, and intuitive tool to help users to maintain or improve their current state. In addition, we have made it easier to create new activities in this platform to help medical staff in charge of rehabilitation and the relatives or friends of users, build their own activities, more easily. We have designed the tools to suit touch screen devices, enabling the user to perform rehabilitation exercises independently and as often as required without additional assistance. In addition, the environment of JClick has been also modified to improve the interaction of people with these problems. Thus, the usability of this application and the activities are much better, getting better experience. Furthermore, previous facts can help to improve the attitude of the rehabilitator, because the work is much easier to do and more interactive and the rehabilitation of the user is improved too, due to having the necessary tools to maintain or improve mental ability.

In the following sections, we explain aspects related to the JClic platform, its architecture, the changes that we will apply to suit touch screen platforms, and the new activity models we will create. We will also discuss the necessary associated usability for this type of work, and outline the related topics that we will carry out to improve the proposal further.

\section{STATE OF ART}

Many authors have written about the stimulation and/or rehabilitation of persons with Alzheimer's through exercises [3]. Such exercises help patients to maintain ability, remain active and to slow down cognitive impairment [4]. In addition, the articles talk about how they can help family members as well as the individual patient, as due to the characteristics of this type of dementia it can cause frustration to all involved. Using this type of rehabilitation can ease this frustration, enabling the patient to overcome shortcomings and make the situation more bearable for those around him.We discuss the following:

- $\quad$ "Ejercicios para potenciar la memoria de los enfermos de Alzheimer" [5]. A help manual, for stimulating the cognitive abilities of patients with dementia. This manual contains illustrated exercises for those who care for a patient.

- "Volver a empezar" [6]. A manual that describes in detail, what the disease is, the process involved, and how to work with each type of patient. It also contains illustrated exercises.

The proposed solutions require a person to be in charge of creating the exercises (photocopy, draw, etc.) and to be at the bedside of the patient continuously to check progress and the realization of said activities. In later sections we will describe how, with the introduction of the new activity models for the 
JClic platform, the work of this person will be easier to perform. In some cases, the patient may be able to do the exercises independently.

\section{JCLIC PLATFORM}

JClic is a platform that consists of a set of free software applications used to perform various types of multimedia educational activities. Among these activities are puzzles, word associations, crosswords, word searches, and text exercises. The exercises use different geometric shapes, images, audio files and other features to make the interaction more visual and entertaining. It is developed in Java [7] programming language, so it can be used on any device that has an operating system: such as Windows, Linus, Mac OS X and Solaris. The activities available in this platform are as follow:

- Simple Association. Consists of two sets of elements, which have to be correctly associated with each other.

- Complex Association. Consists of sets of elements of different sizes, which have to be correctly associated with each other. Some elements in these sets may not be associated with any elements.

- Memory Game. The user has to match the pairs of elements in the panel shown.

- Explore. Displays various elements, and when the user clicks on them, new information appears.

- Identify. Consists of the selection of elements within a set, which meet the described requirements.

- Information Screen. Displays pre-determined information to the user. There may be an option to associate multimedia content.

- Double Puzzle. Consists of two panels, one contains jumbled pieces and the user places them in their correct position in the other panel.

- Exchange Puzzle. In this case, the pieces are disordered in one panel. Through the continuous exchange of the pieces, the user must, find the correct image.

- Hole Puzzle. Consists of a panel, with disordered pieces, with one piece missing. The user must move the pieces into the space left by the missing piece, until he finds the final correct image.

- Text: complete. A piece of text with missing letters, punctuation, or phrases. The user must correctly fill in the missing elements.

- Text: fill in the blanks. Consists of a piece of text with hidden parts that the user corrects. Corrections can be written in the spaces specified, or by selecting the appropriate expressions from a number of options.

- Text: identify. In this activity, the user must click with the mouse to locate words, letters, symbols, numbers, and punctuation.

- Text: order. The user must re-order jumbled up text and paragraphs to the correct position.

- Written answer. For each of the items displayed, the user must enter the requested information correctly.

- Crossword. Consists of completing a panel with definitions that are provided in the form of text, audio, or media.

- Word Search. The user must find the hidden words in a panel of letters.

All the described activities are defined in the abstract, class Activity, as shown in Figure 1.

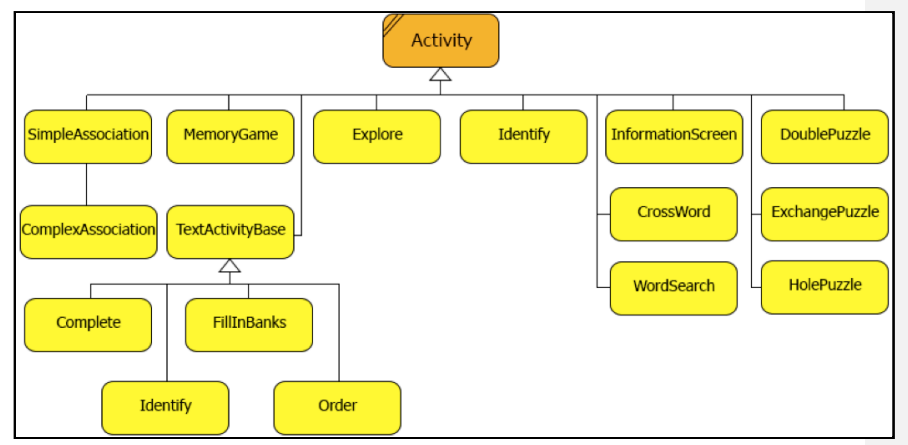

Figure 1. Diagram showing the activities that can be performed

However, this platform is not restricted to these activities, and offers the facility to create customized activities, so we can use it for tasks specified by the developer. Therefore, if we need to include additional activities, we can add to those shown in the abstract class in Figure 1, so that the platform will recognize and run them.

\section{UPDATES TO ADAPT JCLIC TO TOUCH SCREEN DEVICES}

The JClic platform runs activities that use a mouse [2]. In this paper, we propose the use of touch screen devices for people with cognitive problems to facilitate the assimilation of information, which means that we must modify the JClic platform to allow some activities to run in response to touch. Figure 2 shows an example of how the user might respond to the application if we do not adapt its functionality in this way.

a)
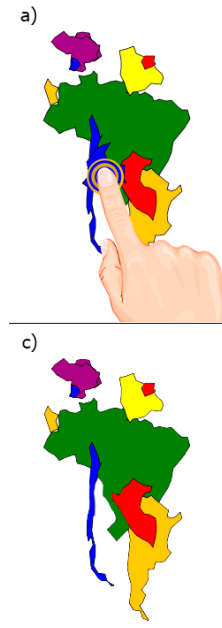

Figure 2. Performing the double puzzle activity on a touchscreen device.
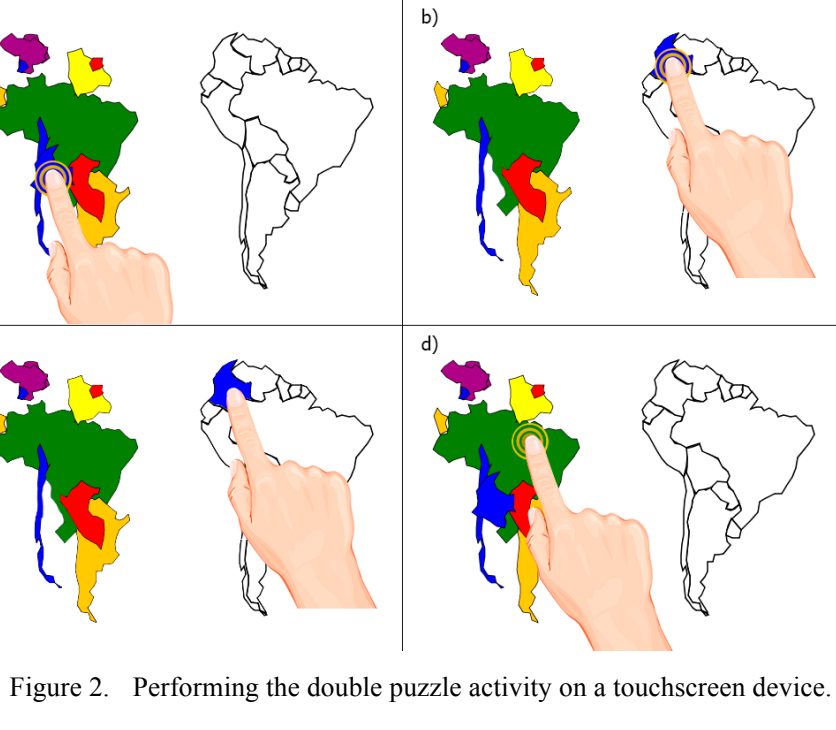
This image shows how, in the "double puzzle activity", the user touches the piece associated with Colombia (a) and drags it across the screen (b) and places it (c). Then the user tries to place another country, but when he points to any other, the piece for Colombia returns to its original position (d). This is because the functionality related to mouse activity is determined by the following process; first, the user clicks on the country he wants to place, and then moves the mouse to the appropriate position and clicks again to place. Once in place, the process is repeated with the other countries. In the illustrated example, when using a finger on the screen, there is no click. It is that click that is responsible for associating the country with its destination, and needs to be made in the unordered panel after putting the finger on the screen.

\section{SYSTEM USABILITY}

Although adaptions were made to facilitate touch screen devices, we need to carry out further updates; otherwise, users may have difficulties using it in this environment become frustrated. This is because the options provided by the environment were designed to be clicked on, and do not require much screen space. However, when a user tries to press the touch screen and uses a finger, the action requires a larger surface area than that of a mouse click, so the interface must be changed, to make the buttons bigger and more accessible. Figure 3 shows an example where the proposed change is observed.

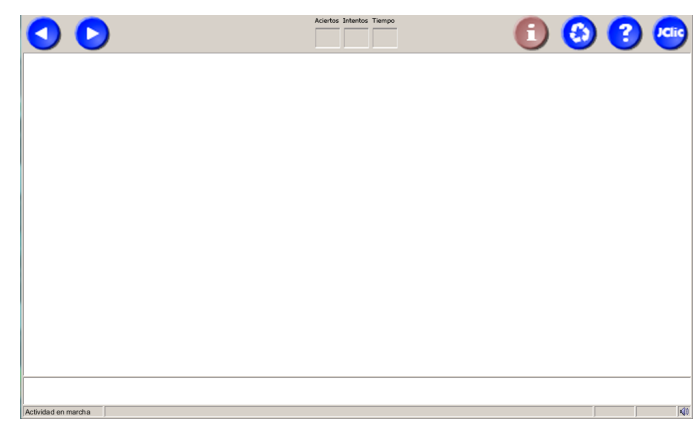

Figure 3. New interface adapted for touch devices.

The size and therefore area of the buttons is sufficiently large so that a finger can press them easily, and the user can focus their attention on the exercises without the difficulty of moving from one activity to another. To create a new type of interface or skin (as it is called within the platform), we need to create an image to show where all its components are laid out, including buttons, information boxes, etc. In addition, we need to associate an $\mathrm{xml}$ file to describe the features of each element, including position and size.

\section{NEW ACTIVITIES AND MODELS}

The proposed package assimilates the experiences of users and experts [5] [6], who have studied and have knowledge of cognitive problems, and new technologies using touch screen devices. Although there are many exercises described in the articles, it is not necessary to create a new activity model for every one of them. Many of the exercises can be adapted to existing activities within the JClic platform. However, we have had to create new models for the exercises, which do not follow a similar structure to those provided in the platform. For this reason, the process described in previous sections has been followed.

Below, we are going to name some of the exercises used in this proposal. A short description of the activity and the model used are also explained. These exercises are shown divided in categories.

\section{A. Memory}

- Related items. We show three objects to the user, and he must select those that are related.

- Example: Drawings of earrings, an ear, and a nose are shown, and the user must select the earrings and the ear.

○ Activity type: Identify

- Select the correct answer. The patient must complete a phrase by choosing the correct elements.

- Example: The user is shown the phrase "the days of the week are..." and must select the correct words from Monday, March, Thursday, September, Wednesday, Saturday, Nephew, Leap, Tuesday, Friday, December, Sunday.

○ Activity type: Identify.

- Draw the template. A geometric figure is displayed for ten seconds. When the image is hidden, the user has to paint the image with his finger. These figures will be simple and drawn within a grid to facilitate the user. For this activity, it is necessary to assist the patient to compare results.

- Example: In the working area of the application, the figure of a boat appears on a grid (Figure 4a). After a set time, the figure disappears and the grid is left blank for the user to draw the boat with a finger (Figure 4b).

- Activity type: This is a new activity model, developed following previously described instructions.

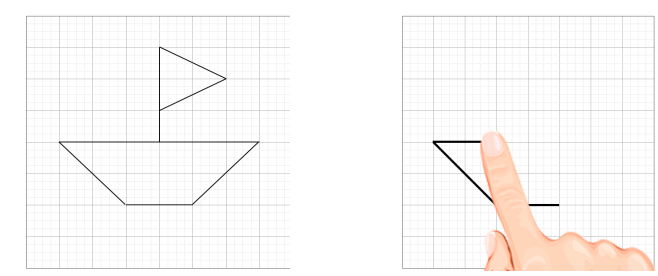

Figure 7a. Grid with a drawing Figure $8 b$. User replicating hidden drawing

\section{B. Attention}

- Select the correct order. The user sees a set of disordered interrelated elements and must put them in the correct order.

- Example: The months of the year are shown out of order, and the user must put them in the correct order. 


\section{○ Activity type: Identify.}

- Divided Attention: The user selects items listed in a panel, and at the same time, a sound is played, likethe sound of a tabletop being hit. These hits continue in a particular sequence and are played one after the other followed by a fixed period of silence. When it is silent, the user identifies the number of hits while also performing another action. Assistance is needed to confirm that the number of hits identified is correct.

- Activity type: Double Identification. This is a new activity model, with a panel similar to the Identify activity and further options relating to the number of hits, the time sequence, and the time between sequences. Another type of sound may replace the hits, Figure 5a and Figure 5b.

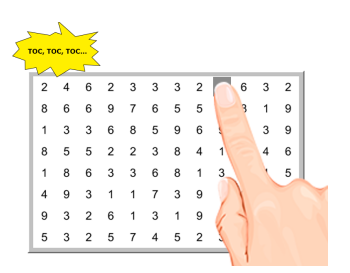

Figure 5a. Double Identification with sounds of hits

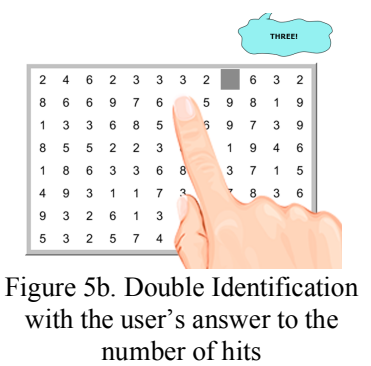
number of hits

\section{Orientation}

- What time? This activity shows a clock with the hands pointing at a particular time. The user must place the clock hands in the position that is the answer to the associated question. For this activity an assistant is required to confirm that the answer is correct, as depending on where the user is, the schedule of determined events may change.

- Example: The clock appears with the hands pointing to ten and ten. The question asked is, "What time is breakfast?" The user moves the dialat the side of the clock to change the position of the hands to point at eight and six and the assistant confirms that the answer is correct.

- Activity type: Clock. This is a new activity with specific options such as the possibility of placing the hands at a certain time and of changing the type of clock.

\section{Gnosias}

- Colours. The user must matchthe name of each colour with the colour itself.

- Example: The names of the colours red, green, blue, yellow, black, pink, white and orange, are listed and samples of the colours themselves are displayed. The patient must match the name to the correct colour.

- Activity type: Simple Association.
- Expressions. A set of images with different expressions is shown alongside a set of names for each expression. The user must associate each expression with the correctimage.

- Example: Several expressions are depicted; sadness, happiness and amazement alongside a panel listing the names. The user must associate a happy face with the name "happiness" and match the other expressions to the correct images.

- Activity type: Complex Association

\section{E. Praxias}

- Connect the dots to complete the image. An image with numbered dots is shown and the user must click on the first dot and join the rest of the dots to complete the image.

- Example: A set of numbered points appears on the screen outlining an image of a duck (Figure 7a). The user connects the dots by passing a finger over them (Figure $7 \mathrm{~b}$ ) until he reaches the end and the user sees the image of the duck

- Activity type: This is a new activity model in which the user determines the location and order of points within the panel.

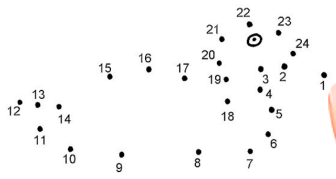

Figure 7a. Image formed with points

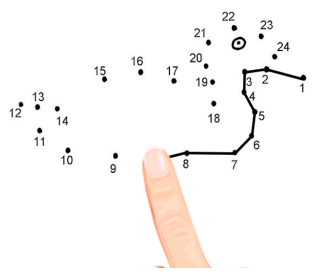

Figure 7b. Points joined with the finger

\section{$F$. Language}

- Begins with. The user is shown a set of letters, and below them, a number of spaces equal to the number of letters required. The user must enter words beginning with the indicated letter a number of predetermined times. This activity requires the assistance of a person to check the results.

- Example: The user is prompted to type ten words that begin with the letters ' $a$ ', 'e', and 'c'. The user selects one of the blank spaces for this purpose and a panel to write in appears, Figure 8a. In the panel, the user writes the words with his finger, Figure $8 \mathrm{~b}$. Once the user has written the word, the panel shrinks back to the space it came from with the word inside it, and the process is repeated, Figure 8c.

- Activity type: Handwriting. A new activity added to the platform. 


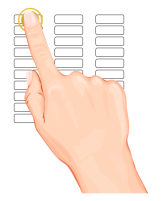

Figure 8a. Blanks for writing manually

\section{airplane}

Figure $8 b$. Writing with the finger

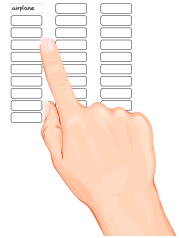

Figure 8c. At the end of the writing, the panel shrinks back to size

\section{G. Calculation}

- Follow the series. This activity consists of a calculation, the result of which is written in the appropriate gaps in a statement to form a sequence.

- Example: The statement shows the following operation: " +4 ". The user starts typing in the first box from the base number. For example, the numbers are, three and seven. So the patient will write $11(7+4), 15,19$, etc., until the spaces are complete and the number 131 is reached.

- Activity type: Series. This is a new activity to determine which operations to perform, using a base number to begin calculating fromin the number of slots available.
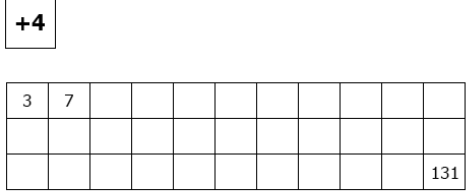

Figure 9. Example of activity Series

- Bingo. A popular game,in which the players have a pre-determined number of boxes displayed in a grid showing random numbers. Each player must cross out the numbers in the boxes, given by the system.

- Example: The user has two opponents and a grid of boxes displaying numbers. The system displays the number seven, which is in the user's box and also in one of his opponents (system) boxes. The user crosses out his number by clicking on it with his finger. The opponent does the same. The game ends when all of the boxes in the game have all their numbers crossed out. If the userdoesn't cross out the system-generated numbers, the game will continue until he has done.

- Activity type: Bingo. New activity model in which we set the number of opponents (difficulty), the time between the numbers displayed, and the number of boxes for the user.

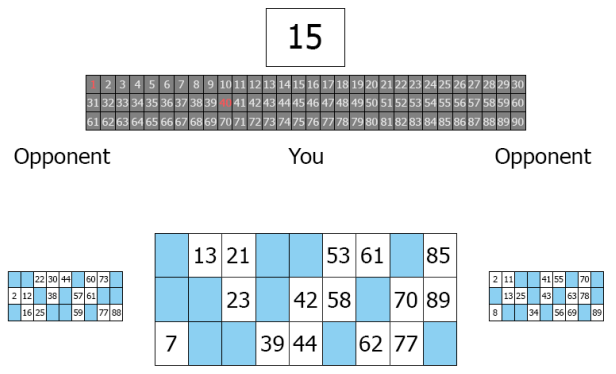

Figure 10. Example of Bingo activity

\section{CONCLUSIONS AND FUTURE WORK}

In this paper, we have remarked on new models and activities created for the JClic platform. It is thanks to these models that we can simplify the task of patient rehabilitation, not just for medical staff, but also for those close to patients. In addition, the process of rehabilitation has been improved and made more enjoyablefor patients with cognitive problems, whether from Alzheimer's or strokes, because the activities and exercises are interactive, more visual and more of them can be carried out independently.

For now, the patients who are developing the sessions have obtained a value $>23$ on the Mini-Mental test [8]. Another test that patients will perform once the above is done, is the minicog test [9]. After completing the test sessions, we will analyze the results statistically with specialist doctors and physicians from the King Abdulaziz Hospital.

However, as the proposals we outline in this paper are our first in this area, there are improvements we are still considering that can improve the platform further, and when complete, the rehabilitation process for such patients will be enhanced. We are considering the following:

- The creation of an algorithm for geometric design, so that when the user draws with his finger, the figures drawn are perfect. With this, we could make a software comparison with the given model.

- The inclusion of voice recognition software so that the user only needs to talk on certain activities instead of typing or selecting the required response by touching the screen.

Our aim is to improvesystem usability and provide better interaction between the user and the touch screen device, and to reduce and/or remove the requirement for additional help from assistants. The patient will be able to perform a greater number of the activities independently, giving them the ability to perform appropriate exercises, and increase the hours spent on rehabilitation.

\section{REFERENCES}

[1] Widmann CN, Beinhoff U, Riepe MW. Everyday memory deficits in very mild Alzheimer's disease. Neurobiol Aging. 2012 Feb;33(2):297303. 
[2] JClic.http://clic.xtec.cat/en/index.htm.(Accessed: January 2013).

[3] Dröes RM, van der Roest HG, van Mierlo L, Meiland FJ. Memory problems in dementia: adaptation and coping strategies and psychosocial treatments. Expert Rev Neurother. 2011 Dec;11(12):1769-81

[4] Hofmann M, Rösler A, Schwarz W, Müller-Spahn F, Kräuchi K, Hock C, Seifritz E. Interactive computer-training as a therapeutic tool in Alzheimer's disease. Compr Psychiatry. 2003 May-Jun;44(3):213-9

[5] Pedro Gil, Raquel Yubero, Llanos Morón. Ejercicios para potenciar la memoria de los enfermos de Alzheimer (Exercises to enhance the memory on patients of Alzheimer). Unidad de Memoria. Servicio de Geriatría del Hospital Universitario San Carlos (Madrid). Editorial Just in Time S.L.
[6] L. Tárraga y M. Boada (eds.). Volver a empezar. Ejercicios prácticos de estimulación cognitiva para enfermos de Alzheimer. (Back to the start. Practice exercises for a cognitive stimulation on patients of Alzheimer).

[7] Java. http://www.java.com/en/. (Accessed: January 2013)M.F. Folstein, S.E. Folstein and P.R. Mchugh, "Mini-Mental State: a practical method for grading the cognitive state of patients for the clinicians," J Psychiatr Res, vol. 12, pp. 189-198, 1975.

[8] Borson S, Scanlan J, Brush M, Vitaliano P, Dokmak A. The mini-cog: a cognitive'vital signs' measure for dementia screening in multi-lingual elderly. Int JGeriatr Psychiatry. 2000 Nov;15(11):1021-7 